\title{
Crack Detection Using Fluxgate Magnetic Field Sensor
}

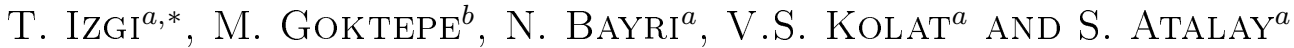 \\ ${ }^{a}$ Inonu University Science and Art Faculty, Physics Department, 44069 Malatya, Turkey \\ ${ }^{b}$ Balikesir University, Faculty of Science and Literature, Department of Physics, 10145 Balikesir, Turkey \\ In this study, the variation of the magnetic flux distribution in a magnetised ferromagnetic material which has \\ in homogeneity as a crack is studied. An orthogonal fluxgate magnetic field sensor was used in the inspection of \\ cracks. In the fluxgate sensor, the sensing element $\left(\mathrm{Co}_{0.94} \mathrm{Fe}_{0.06}\right)_{72.5} \mathrm{Si}_{12.5} \mathrm{~B}_{15}$ amorphous ferromagnetic wire was \\ placed inside a pickup coil winding with $50 \mu \mathrm{m}$ copper wire and connected to a signal generator and the output \\ from pick-up coil was detected using a lock-in amplifier. The surface profile of magnetic materials with a crack was \\ obtained using a specially designed 3-dimensional moving system. A large decrease in the output voltage of the \\ sensor was observed when the sensor was moved on the top of the crack, after the further movement of the sensor \\ the output voltage came back to the previous value.
}

DOI: $10.12693 /$ APhysPolA.125.211

PACS: 85.75.Ss, $46.50 .+\mathrm{a}, 75.50 . \mathrm{Kj}$

\section{Introduction}

Non-destructive evaluation systems have been widely utilized for inspection of many materials used in safety and critical applications. Magnetic non-destructive testing methods such as the magnetic flux leakage (MFL) method, eddy current testing, and the residual magnetic field technique are useful methods for the prevention of accidents due to break of mechanical parts in the machines, and also useful for the prolongation of the service life-time of a structure [1].

The magnetic flux leakage (MFL) method is the most common and cost-effective nondestructive magnetic testing technique used in various nondestructive testing applications. This method is based on measuring the magnetic leakage field over the surface of a test specimen in the vicinity of small defects such as cracks [2]. In the measurement of MFL, we need to visualize the magnetic map of the surface with good precision and sensitivity.

The most commonly used sensors for these applications are fluxgate sensor, the Hall effect sensor and induction coil sensor [3-5]. Related to recent advances in magnetic sensor technology, for the detection of very small changes in the MFL distribution, high resolution magnetic sensors such as SQUID [3], GMR [3], and GMI [6-8] have been introduced.

The microstructural changes induced in a thermal or stressed environment and cracked region of industrial applications and moving machine parts are often degradation of the mechanical properties of steel. The fluxgate sensor can be used either to detect magnetic fields created by current passing through conductors or to detect localised magnetic fields non-destructive testing applications. The occurred discontinuity resulting from a crack also produces disturbance in the magnetic field in the material, and the magnitude of the disturbance is deter-

\footnotetext{
*corresponding author
}

mined by the size and shape of the crack. The fluxgate magnetic field sensor with amorphous ferromagnetic core can be to capture cracked regions in the materials.

\section{Experimental}

The basic configuration of the fluxgate sensor is shown in Fig. 1a. The sensing element consists of the soft magnetic amorphous ferromagnetic $\left(\mathrm{Co}_{0.94} \mathrm{Fe}_{0.06}\right)_{72.5} \mathrm{Si}_{12.5} \mathrm{~B}_{15}$ core and pick-up coil. Amorphous wire was annealed at $460^{\circ} \mathrm{C}$ for $90 \mathrm{~min}$. The presented fluxgate sensor is orthogonal type. The magnetic wire is excited by an $\mathrm{AC}$ sine wave with $50 \mathrm{kHz}$ frequency and wire was magnetically saturated in the circumferential direction. The magnetic field produced by AC sine wave should be large enough to saturate the core. The second harmonic of induced voltage in the pick-up coil was measured using a lock-in amplifier. The sensor was placed in a shielded solenoid and external magnetic field

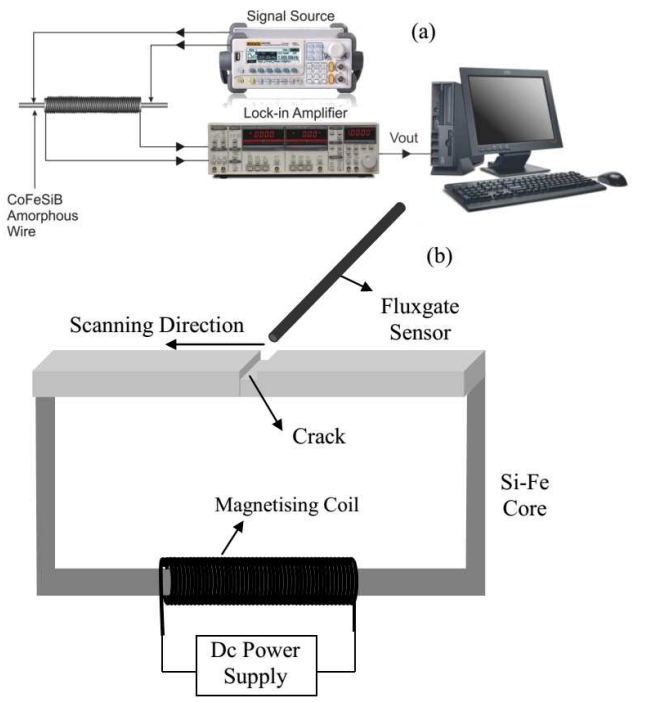

Fig. 1. The basic configuration of (a) the fluxgate sensor and (b) experimental setup. 


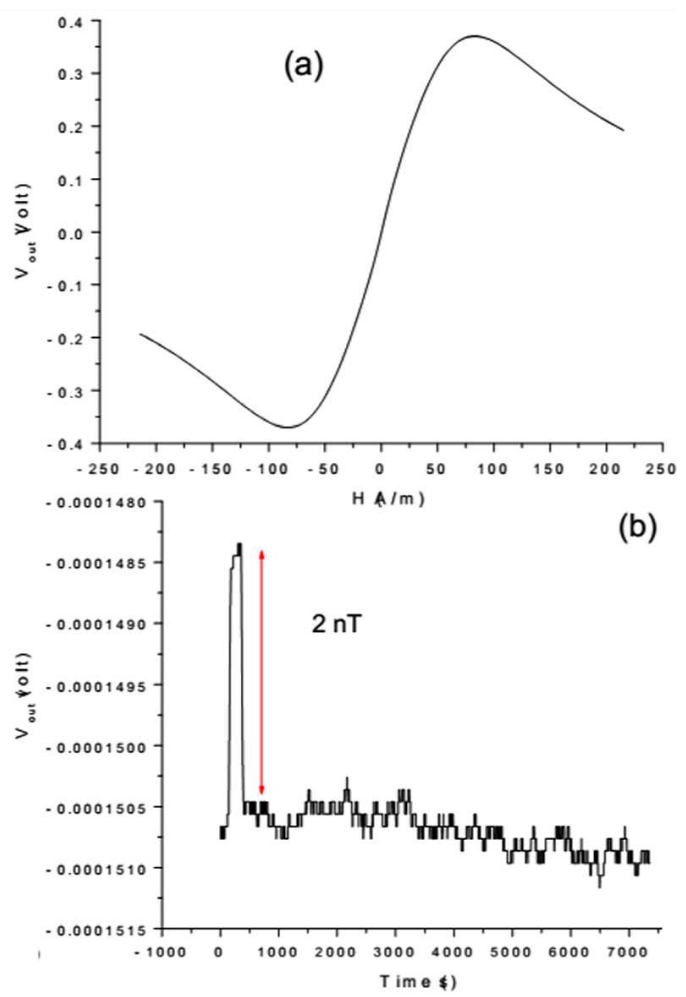

Fig. 2. Sensor output as a function of external magnetic field, (b) sensor output as a function of time.

applied along the sample length. The magnetic field was applied using a bipolar power supply-solenoid system.

The variation of sample output voltage measured from lock-in as a function of external field is given in Fig. 2. The sensor shows a nearly linear change at $\pm 50 \mathrm{~A} / \mathrm{m}$ magnetic field regions. $2 \mathrm{nT}$ magnetic field pulse was applied to sensor and the change in the output can be seen in Fig. 2b. It can be seen that the typical sensitivity of fluxgate sensor is better than $1 \mathrm{nT}$.

\section{Results and discussion}

Figure 3 shows magnetic map of a small magnet with $1.5 \times 1.5 \mathrm{~mm}^{2}$ size. Sensor was placed orthogonally to magnet and scanned with $0.1 \mathrm{~mm}$ steps. A large increase in the sensor output was observed on the top of magnet, Fig. 3b is the contour plot of Fig. 3a.

An experimental setup has been used to capture cracks in a material as shown in Fig. 1b. C-core was used to magnetize the system. C-core was made from $3 \% \mathrm{Si}-\mathrm{Fe}$ laminations with $110 \mathrm{~mm}$ limb length with $1200 \mathrm{~mm}^{2}$ cross-sectional area of limbs and $220 \mathrm{~mm}$ distance between the limbs. $N=110$ turn magnetisation coil was made on the $\mathrm{C}$-core to get magnetisation in the core.

Number of crystalline $3 \% \mathrm{Si}-\mathrm{Fe}$ lamination sheets have been used to simulate cracks in the material. Lamination sheets with $0.35 \mathrm{~mm}$ thickness, $30 \mathrm{~mm}$ width, and $150 \mathrm{~mm}$ length have been stacked on each other to simulate full and half cracks. $1.6 \mathrm{~mm}$ wide crack has been made in the middle of the sample and the crack was located along the sample of the cross-section as a full crack.
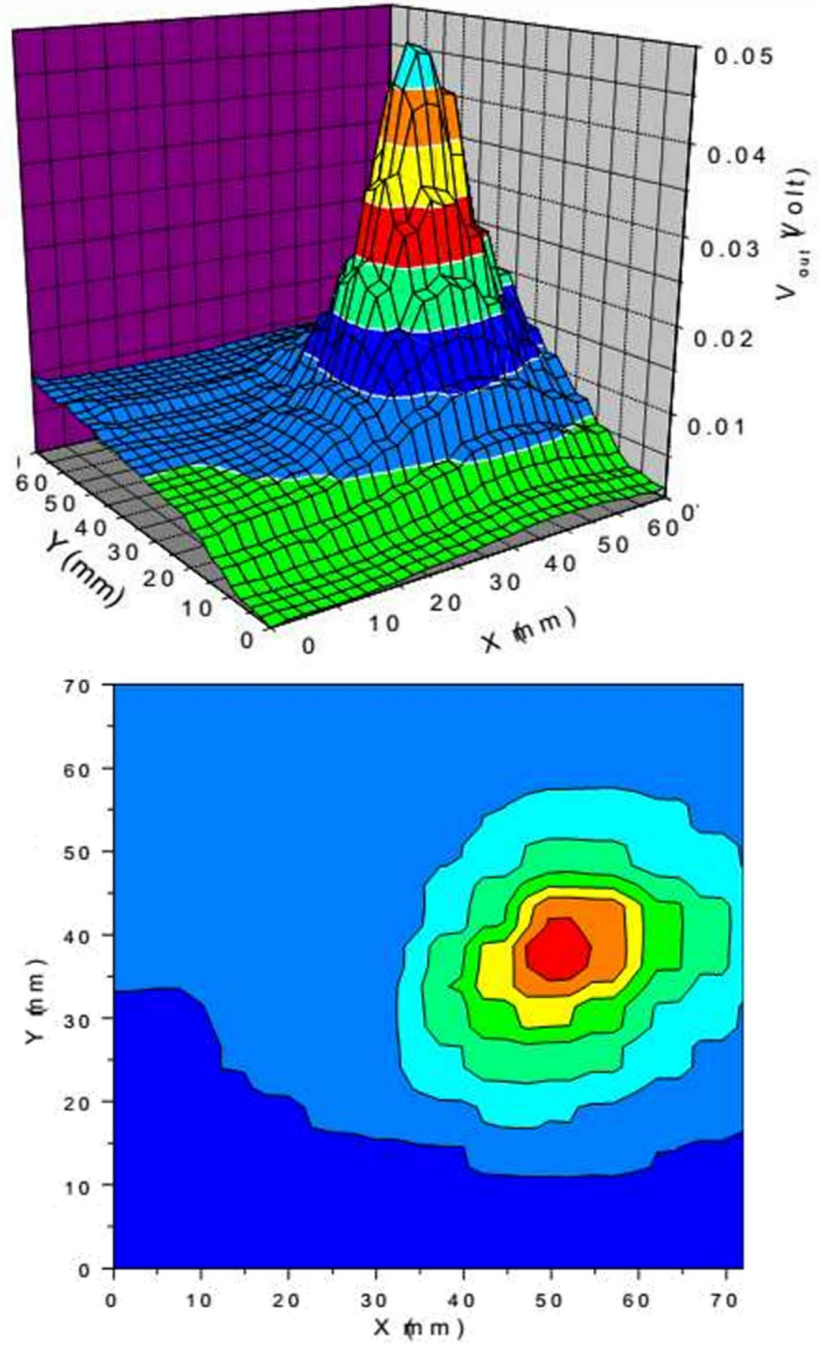

Fig. 3. Magnetic mapping of a small magnet.

$2 \mathrm{~mm}$ wide and $1.6 \mathrm{~mm}$ deep crack is also prepared in the middle of the sample and called as a half crack as shown in Fig. 1b. C-core was magnetised by applying an dc current to the 100 turn magnetisation windings. Therefore, a dc magnetisation occurred in the core because of the reorientation of the magnetic domains along the flux lines.

During this progress variation of flux density $(\mathrm{d} B / \mathrm{d} t \neq 0)$ in time becomes greater than zero. Magnetic flux lines follow closed magnetic circuit than jump to the sample from the C-core limbs and flux flows in the core up to meet a crack. The normal component of the flux gets bigger around the cracked region. An amorphous wire has been used to capture normal component of magnetic flux lines.

The amplitude of the measured signal was changed when amorphous wire captured the normal component of the magnetic flux on the surface of the magnetised sample and that means a crack or scratch is located around the wire. When a crack or a scratch somehow occurs on 
the material, a discontinuity suddenly appears and normal component of magnetic flux becomes greater than zero instead of a signal becoming big enough to occur in the amorphous wire. Therefore, a signal variation could be read from the lock-in amplifier.
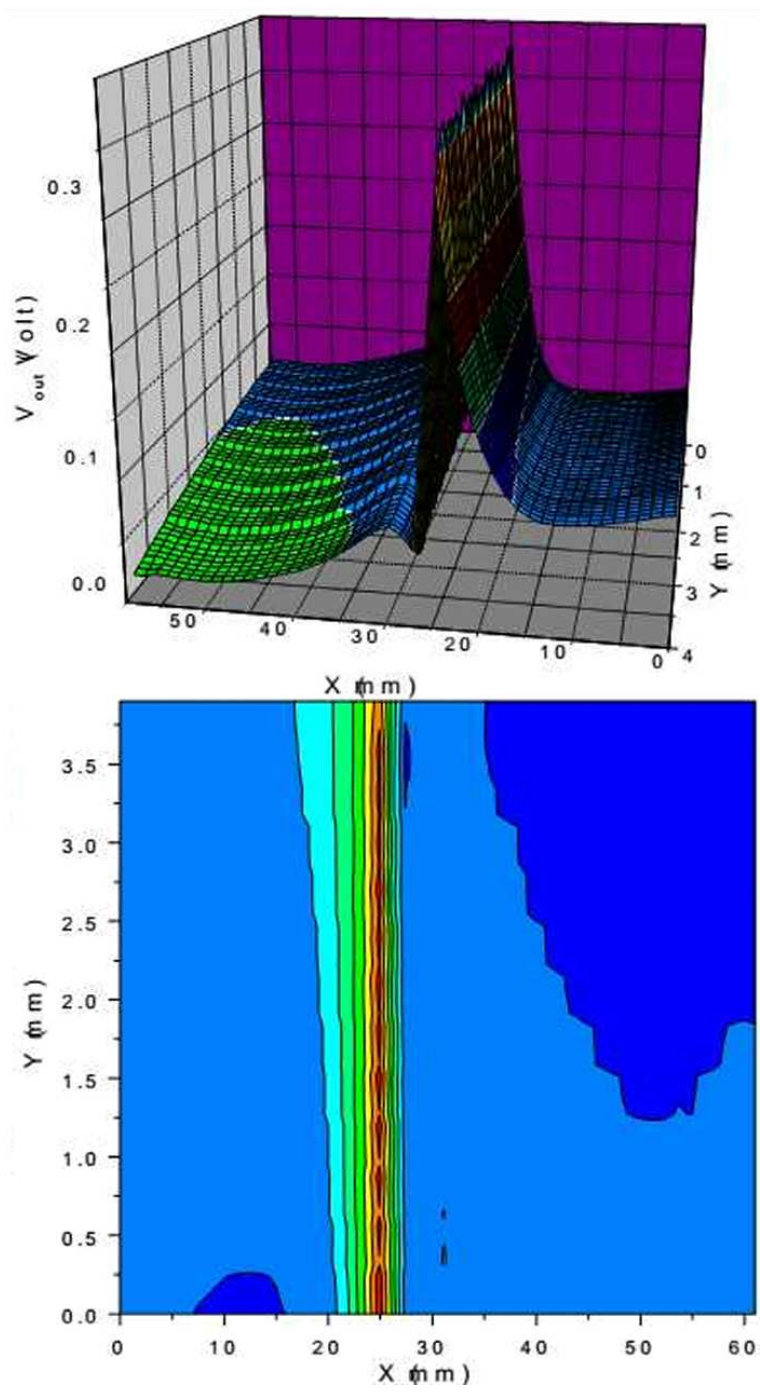

Fig. 4. Variation of sensor signal around crack region.
A large decrease in the output voltage of the sensor circuit was observed when the sensor was moved on the top of the crack, after the further movement of the sensor the output voltage came back to the nearly previous value (Fig. 4). If the magnetised materials have not got any crack, no significant variation in the sensor output was measured, because all magnetic flux lines pass through the material due to its higher permeability.

\section{Conclusion}

In conclusion, results have shown that there is a good correlation between the size and position of a crack and the sensor output. This method has great potential for application in the inspection of cracks in the surface or inside of magnetic materials.

\section{Acknowledgments}

This work was supported by Inonu University Research Fund with the project number 2012/168.

\section{References}

[1] J.G. Martin, J. Gomez-Gil, E.V. Sanchez, Sensors 11, 2525 (2011).

[2] K. Tsukada, M. Yoshioka, T. Kiwa, Y. Hirano, NDTEE Int. 44, 101 (2011).

[3] J.E. Lenz, Proc. IEEE 78, 973 (1990).

[4] M. Butta, I. Sasada, J. Appl. Phys. 111, p07E517 (2012).

[5] S. Atalay, N. Bayri, T. Izgi, F.E. Atalay, V.S. Kolat, Sens. Actuat. A, Phys. 158, 37 (2010).

[6] M.M. Tehranchi, M. Ranjbaran, H. Eftekhari, Sens. Actuat. A, Phys. 170, 55 (2011).

[7] G.V. Kurlyandskaya, D. de Cos, S.O. Volchkov, Russ. J. Nondestruct. Test. 45, 377 (2009).

[8] M. Goktepe, Y. Ege, N. Bayri, S. Atalay, Phys. Status Solidi C 12, 3436 (2004). 